\title{
Precision predictions for associated gluino-gaugino production at the LHC
}

\section{Benjamin Fuks*}

Sorbonne Universités, UPMC Univ. Paris 06, UMR 7589, LPTHE, F-75005 Paris, France CNRS, UMR 7589, LPTHE, F-75005 Paris, France

Institut Universitaire de France, 103 boulevard Saint-Michel, F-75005 Paris, France

E-mail: fukselpthe.jussieu.fr

\section{Michael Klasen ${ }^{\dagger} \ddagger$}

Institut für Theoretische Physik, Westfälische Wilhelms-Universität Münster,

Wilhelm-Klemm-Straße 9, D-48149 Münster, Germany

E-mail: michael.klasen@uni-muenster.de

\section{Marthijn Sunder ${ }^{\S}$}

Institut für Theoretische Physik, Westfälische Wilhelms-Universität Münster, Wilhelm-Klemm-Straße 9, D-48149 Münster, Germany

E-mail: mpasunder@uni-muenster.de

\begin{abstract}
Now that the mass limits for gluinos have been pushed to the few-TeV range, they might only be visible at the LHC in associated production with lighter gauginos. We compute the corresponding cross section at next-to-leading logarithmic (NLL) and next-to-leading order (NLO) precision in the QCD coupling constant. The resulting expressions are implemented in the public code RESUMMINO and can be directly used in the corresponding experimental searches.
\end{abstract}

EPS-HEP 2017, European Physical Society conference on High Energy Physics

5-12 July 2017

Venice, Italy

\footnotetext{
* Supported by CNRS under contract PICS 150423 and Théorie-LHC-France initiative of CNRS (IN2P3/INP). † Speaker.

¥ Supported by BMBF under contract 05H15PMCCA.

${ }^{\S}$ Supported by DFG under contract GRK 2149.
} 


\section{Cross sections for supersymmetric particles at the LHC}

Supersymmetry (SUSY), a long-standing, well-motivated and complete extension of the Standard Model (SM) of particle physics with a rich phenomenology, continues to be searched for at CERN's Large Hadron Collider (LHC). For many years, squarks and gluinos have been at the centre of this search due to their strong interactions and correspondingly large cross sections. However, the mass limits for these particles are now already in the few- $\mathrm{TeV}$ range, so that pair production of these particles might soon get out of reach at LHC energies of 13 or $14 \mathrm{TeV}$. Associated production of squarks and gluinos with an electroweak superpartner (a gaugino or higgsino, or equivalently a neutralino or chargino), that is still allowed to be light and that is motivated for its part by dark matter observations, might then be the only possibility to study them. The corresponding cross sections, which are, like the average produced final-state mass, of intermediate size and have been known for many years at next-to-leading order (NLO) of QCD, should then be known with the same up-to-date precision as those for strong and weak SUSY particle pair production, i.e. at nextto-leading logarithmic (NLL) accuracy [1] and beyond [2], so that they can be used by the ATLAS [3] and CMS [4] collaborations.

\section{Analytical results}

The tree-level Feynman diagrams for associated gluino-gaugino production are shown in Fig. 1.

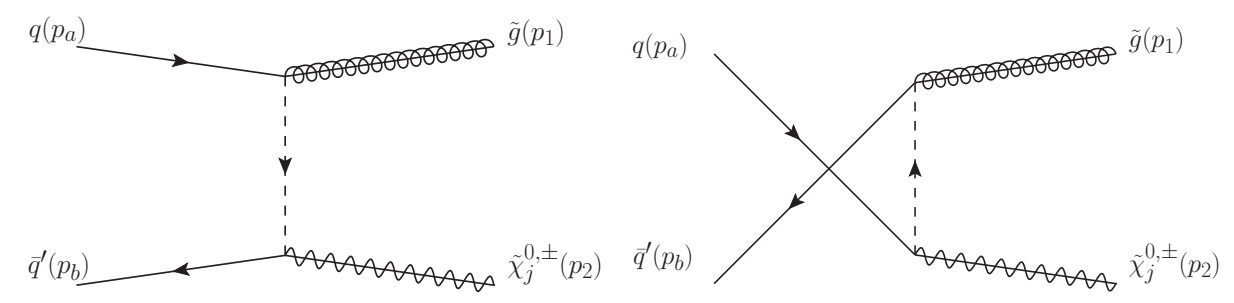

Figure 1: Tree-level Feynman diagrams for associated gluino-gaugino production.

They lead to the squared matrix elements

$$
\begin{aligned}
\mathscr{M}_{t} \mathscr{M}_{t_{c}}^{*} & =\frac{C_{A} C_{F} e g_{s}\left(\mu_{r}\right)}{\left(m_{\tilde{q}}^{2}-t\right)\left(m_{\tilde{q}_{c}}^{2}-t\right)}\left(\mathscr{L}^{\prime} \mathscr{L}_{c}^{\prime}+\mathscr{R}^{\prime} \mathscr{R}_{c}^{\prime}\right)\left(L L_{c}+R R_{c}\right)\left(m_{\tilde{g}}^{2}-t\right)\left(m_{\tilde{\chi}}^{2}-t\right), \\
\mathscr{M}_{u} \mathscr{M}_{u_{c}}^{*} & =\frac{C_{A} C_{F} e g_{s}\left(\mu_{r}\right)}{\left(m_{\tilde{q}}^{2}-u\right)\left(m_{\tilde{q}_{c}}^{2}-u\right)}\left(\mathscr{L} \mathscr{L}_{c}+\mathscr{R}_{\mathscr{R}_{c}}\right)\left(L^{\prime} L_{c}^{\prime}+R^{\prime} R_{c}^{\prime}\right)\left(m_{\tilde{g}}^{2}-u\right)\left(m_{\tilde{\chi}}^{2}-u\right), \\
\mathscr{M}_{t} \mathscr{M}_{u_{c}}^{*} & =\frac{C_{A} C_{F} e g_{s}\left(\mu_{r}\right)}{\left(m_{\tilde{q}}^{2}-t\right)\left(m_{\tilde{q}_{c}}^{2}-u\right)}\left[\left(-s^{2}+t^{2}+u^{2}+\left(m_{\tilde{\chi}}^{2}+m_{\tilde{g}}^{2}\right)(s-t-u)+2 m_{\tilde{g}}^{2} m_{\tilde{\chi}}^{2}\right)\right. \\
& \left.\times\left(L L_{c} \mathscr{L}^{\prime} \mathscr{L}_{c}^{\prime}+R R_{c} \mathscr{R}^{\prime} \mathscr{R}_{c}^{\prime}\right)+2 m_{\tilde{g}} m_{\tilde{\chi}} s\left(\mathscr{R} R_{c} \mathscr{L}^{\prime} L_{c}^{\prime}+\mathscr{L}_{c} \mathscr{R}^{\prime} R_{c}^{\prime}\right)\right],
\end{aligned}
$$

and consequently the partonic and hadronic cross sections

$$
\mathrm{d} \sigma_{a b}^{(0)}=\int_{2} \mathrm{~d} \sigma^{B}=\int \frac{1}{2 s} \frac{1}{4 C_{A}^{2}} \sum_{\tilde{q}, \tilde{q}_{c}}\left(\mathscr{M}_{t} \mathscr{M}_{t_{c}}^{*}+\mathscr{M}_{u} \mathscr{M}_{u_{c}}^{*}-2 \operatorname{Re}\left(\mathscr{M}_{t} \mathscr{M}_{u_{c}}^{*}\right)\right) \mathrm{dPS}(2)
$$


and

$$
\begin{aligned}
\sigma_{A B}=\int M^{2} \frac{\mathrm{d} \sigma_{A B}}{\mathrm{~d} M^{2}}(\tau) & =\sum_{a, b} \int_{0}^{1} \mathrm{~d} x_{a} \mathrm{~d} x_{b} \mathrm{~d} z\left[x_{a} f_{a / A}\left(x_{a}, \mu_{f}^{2}\right)\right]\left[x_{b} f_{b / B}\left(x_{b}, \mu_{f}^{2}\right)\right] \\
& \times\left[z \mathrm{~d} \sigma_{a b}\left(z, M^{2}, \mu_{r}^{2}, \mu_{f}^{2}\right)\right] \delta\left(\tau-x_{a} x_{b} z\right)
\end{aligned}
$$

Here $C_{A, F}$ denote QCD colour factors, $e, g_{s}\left(\mu_{r}\right)$ electromagnetic and (scale-dependent) strong couplings, $s, t, u$ Mandelstam variables, $\operatorname{dPS}^{(2)}$ the two-particle phase space, $L, R$ and $\mathscr{L}, \mathscr{R}$ gaugino and gluino coupling strengths, and $\tau=M^{2} / S$ the ratio of the squared invariant mass of the produced SUSY particle pair to the hadronic centre-of-mass energy. The NLO corrections are computed with the Catani-Seymour dipole subtraction method [5]

$$
\mathrm{d} \sigma_{a b}^{(1)}=\sigma^{\{3\}}+\sigma^{\{2\}}+\sigma^{C}=\int_{3}\left[\mathrm{~d} \sigma^{R}-\mathrm{d} \sigma^{A}\right]_{\varepsilon=0}+\int_{2}\left[\mathrm{~d} \sigma^{V}+\int_{1} \mathrm{~d} \sigma^{A}\right]_{\varepsilon=0}+\sigma^{C}
$$

in $D=4-2 \varepsilon$ dimensions, and the final result agrees with our previous calculation [6].

Close to partonic threshold, when $z=\frac{M^{2}}{s} \rightarrow 1$, large logarithms $\left(\frac{\alpha_{s}}{2 \pi}\right)^{n}\left[\frac{\ln ^{m}(1-z)}{1-z}\right]_{+}$spoil the convergence of the perturbative series and must be resummed to all orders. This is most easily achieved in Mellin space, where the resummed cross section

$$
\mathrm{d} \sigma_{a b \rightarrow i j}^{(\text {res. })}\left(N, M^{2}, \mu^{2}\right)=\sum_{I} \mathscr{H}_{a b \rightarrow i j, I}\left(M^{2}, \mu^{2}\right) \Delta_{a}\left(N, M^{2}, \mu^{2}\right) \Delta_{b}\left(N, M^{2}, \mu^{2}\right) \Delta_{a b \rightarrow i j, I}\left(N, M^{2}, \mu^{2}\right)
$$

factorises into soft-collinear and soft functions

$$
\Delta_{a} \Delta_{b} \Delta_{a b \rightarrow i j, I}=\exp \left[L G_{a b}^{(1)}(\lambda)+G_{a b \rightarrow i j, I}^{(2)}\left(\lambda, M^{2} / \mu^{2}\right)+\ldots\right]
$$

with leading and next-to-leading logarithms [7]

$$
\begin{aligned}
G_{a b}^{(1)}(\lambda) & =g_{a}^{(1)}(\lambda)+g_{b}^{(1)}(\lambda), \\
G_{a b \rightarrow i j}^{(2)}(\lambda) & =g_{a}^{(2)}\left(\lambda, M^{2}, \mu_{r}^{2}, \mu_{f}^{2}\right)+g_{b}^{(2)}\left(\lambda, M^{2}, \mu_{r}^{2}, \mu_{f}^{2}\right)+h_{a b \rightarrow i j, I}^{(2)}(\lambda) .
\end{aligned}
$$

The soft anomalous dimension $h_{a b \rightarrow i j, I}^{(2)}$ and hard matching coefficient $\mathscr{H}_{a b \rightarrow i j, I}$ are process-dependent. The former reads in the present case

$$
h_{a b \rightarrow i j, I}^{(2)}(\lambda)=\frac{2 \pi}{\alpha_{s}} \frac{\ln (1-2 \lambda)}{2 \beta_{0}} \operatorname{Re}\left\{\frac{\alpha_{s}}{2 \pi} C_{A}\left[\ln 2+i \pi-1+\ln \left(\frac{m_{\tilde{g}}^{2}-t}{\sqrt{2} m_{\tilde{g}} \sqrt{s}}\right)+\ln \left(\frac{m_{\tilde{g}}^{2}-u}{\sqrt{2} m_{\tilde{g}} \sqrt{s}}\right)\right]\right\},
$$

while the latter $\left(\mathscr{H}_{a b \rightarrow i j, I}\right)$ can be found in Ref. [8].

\section{Numerical results}

We study the impact of the NLL+NLO corrections in a phenomenological Minimal SUSY SM with 13 free parameters (pMSSM-13). Input parameters are in particular the bino and gluino soft SUSY-breaking masses $M_{1}$ and $M_{3}$, with the wino mass fixed to $M_{2} \simeq 2 M_{1}$. The physical mass spectrum is then obtained with SPheno 3.37 [9] and shown for our example scenario in Fig. 2. Fig. 3 shows the invariant-mass distribution of the produced sparticles. Additional radiation shifts 


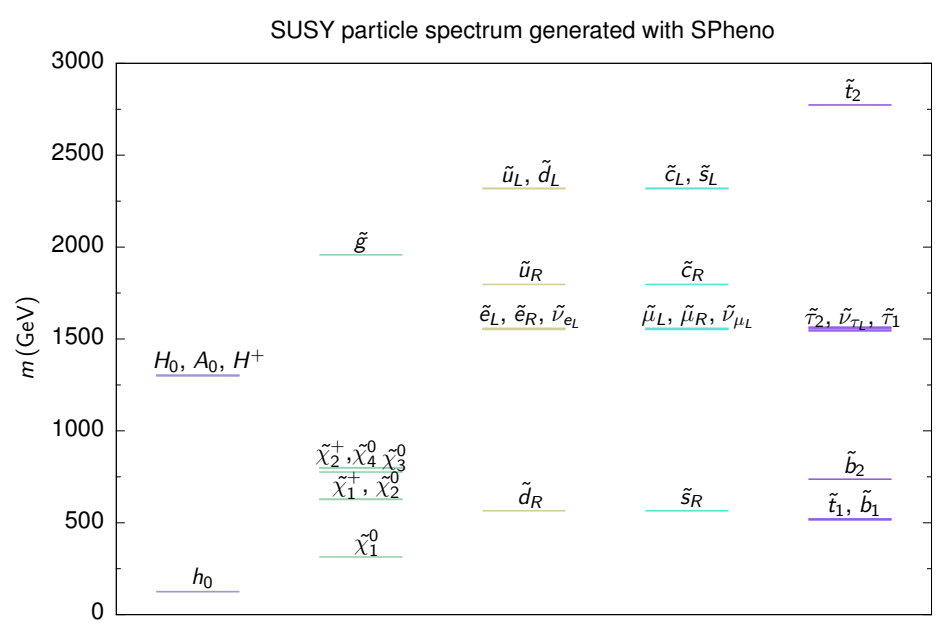

Figure 2: Default pMSSM-13 scenario with light gauginos, a heavy gluino and the correct mass of the lightest Higgs boson.

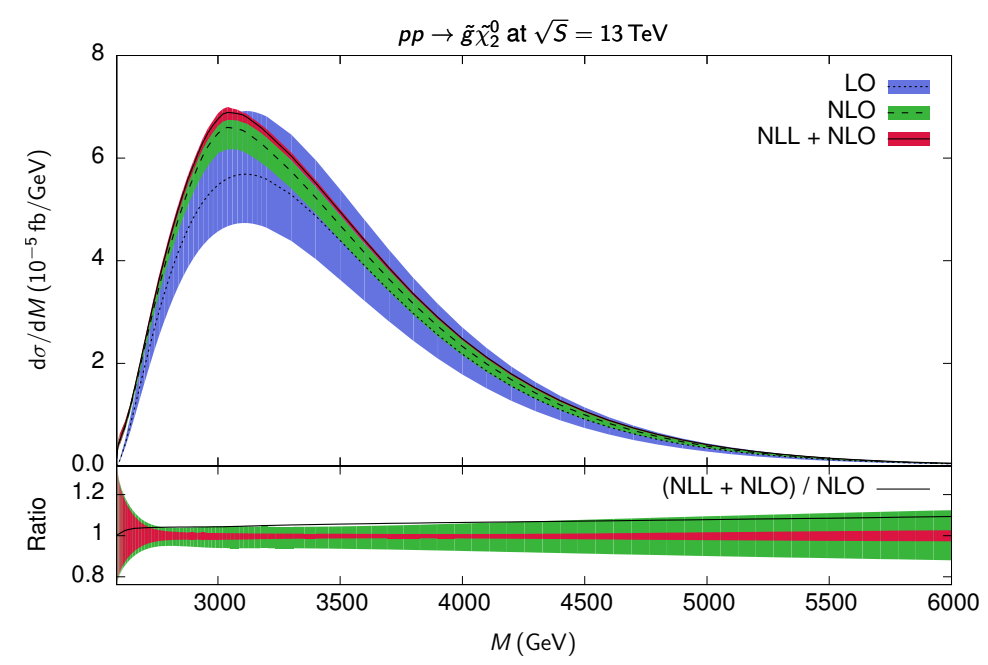

Figure 3: Invariant-mass distributions in LO, NLO and NLL+NLO at the LHC with its current centre-ofmass energy of $13 \mathrm{TeV}$.

the maximum to lower invariant masses and reduces the scale dependence. At large $M$, the NLL corrections increase the NLO cross section by up to $10 \%$, and the total scale dependence is reduced there from $30 \%$ to $5 \%$ (lower panel). The variation of the gluino mass in Fig. 4 demonstrates that associated gluino-gaugino pairs in this class of SUSY scenarios will soon be observable at the LHC with an integrated luminosity of $100 \mathrm{fb}^{-1}$ up to gluino masses of $3 \mathrm{TeV}$.

\section{Conclusion}

The semi-weak associated production of gluinos and gauginos might soon become relevant, if 


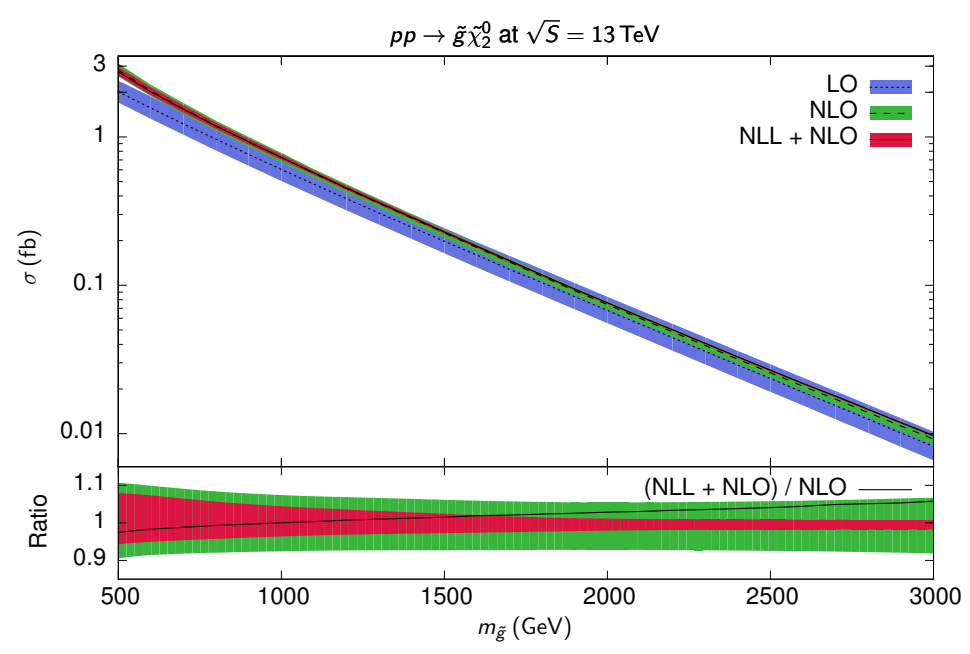

Figure 4: Total cross section as a function of the gluino mass in LO, NLO and NLL+NLO.

gluinos are too heavy to be pair-produced at the LHC. This would indeed be theoretically expected from the GUT relation $M_{1}=M_{2} / 2=M_{3} / 6$ among the soft SUSY-breaking gaugino and gluino mass parameters. We have summarised the analytical calculations of the process-dependent pieces of a threshold-resummation calculation at NLL accuracy, i.e. of the soft anomalous dimension and the hard matching coefficient, before matching the result to a full NLO calculation and performing numerically an inverse Mellin transform. As we have seen, the NLL contributions can increase the NLO invariant mass distribution by up to $10 \%$ and reduce the total scale dependence from $30 \%$ to $5 \%$. The parton density function (PDF) uncertainty should be reduced by including thresholdimproved PDFs in the near future.

\section{References}

[1] B. Fuks, M. Klasen, D. R. Lamprea and M. Rothering, Eur. Phys. J. C 73 (2013) 2480; see also JHEP 1401 (2014) 168, JHEP 1210 (2012) 081, Nucl. Phys. B 849 (2011) 64, Nucl. Phys. B 842 (2011) 51, Phys. Lett. B 688 (2010) 208, Nucl. Phys. B 794 (2008) 46, Nucl. Phys. B 777 (2007) 157, and Phys. Rev. D 74 (2006) 015001.

[2] W. Beenakker, C. Borschensky, M. Krämer, A. Kulesza and E. Laenen, JHEP 1612 (2016) 133

[3] G. Aad et al. [ATLAS Collaboration], Phys. Rev. D 93 (2016) 052002.

[4] V. Khachatryan et al. [CMS Collaboration], Eur. Phys. J. C 74 (2014) 3036.

[5] S. Catani, S. Dittmaier, M. H. Seymour and Z. Trocsanyi, Nucl. Phys. B 627 (2002) 189.

[6] E. L. Berger, M. Klasen and T. M. P. Tait, Phys. Rev. D 62 (2000) 095014; see also Phys. Lett. B 459 (1999) 165. and M. Spira, hep-ph/0211145.

[7] S. Catani, D. de Florian, M. Grazzini and P. Nason, JHEP 0307 (2003) 028.

[8] B. Fuks, M. Klasen and M. Rothering, JHEP 1607 (2016) 053;

M. Rothering, PhD thesis, WWU Münster, 2016.

[9] W. Porod and F. Staub, Comput. Phys. Commun. 183 (2012) 2458. 